\title{
Transition from lateral to transverse phase separation during film co-deposition
}

\author{
C. D. Adams \\ Department of Materials Science and Engineering, University of Michigan, Ann Arbor, Michigan 48109 \\ M. Atzmon \\ Department of Nuclear Engineering, University of Michigan, Ann Arbor, Michigan 48109 \\ Y.-T. Cheng \\ General Motors Research Laboratories, Physical Chemistry Dept., Warren, Michigan 48090-9055 \\ D. J. Srolovitz \\ Department of Materials Science and Engineering, University of Michigan, Ann Arbor, Michigan 48109
}

(Received 22 April 1991; accepted for publication 13 August 1991)

\begin{abstract}
We report observations of two distinct types of phase-separated microstructures in codeposited Al-Ge films. In the initial stages of growth, lateral phase separation is observed, with a temperature dependence consistent with surface diffusion. As the film grows thicker, the Ge-rich phase becomes increasingly buried, and a transverse phase-separated microstructure results, consisting of an Al-rich layer covering a Ge-rich layer. This observation is explained in terms of the competition between surface and interfacial free energies. We discuss the kinetic aspects of the phase separation process, and the resulting behavior in the thick-film limit.
\end{abstract}

Two-phase films are employed in a variety of applications, including magnetism, superconductivity, and catalysis. While controlled multilayer deposition is one possible method of synthesis commonly used for electronic materials, in situ phase separation during deposition is an attractive approach when a precise superlattice structure is not required. An obvious choice for such a process would be an immiscible binary system. Given adequate kinetic conditions, phase separation is expected to occur during co-deposition.

We have recently reported ${ }^{1,2}$ on the surface morphology and microstructure of co-deposited Al-Ge films of thickness 100-200 $\mathrm{nm}$. The Al-Ge system has a simple eutectic phase diagram ${ }^{3}$ with small mutual solubilities, and is thus expected to exhibit phase separation. We observed phase separation within the plane of the film with a length scale which increased with temperature. The phase separation process was modeled by a surface-diffusion mechanism, and the asymptotic, steady-state, domain diameter $R_{\infty}$ was shown to be proportional to $\sqrt{\widetilde{D}_{s} \delta / v}$, where $\widetilde{D}_{s}$ is the surface interdiffusion coefficient, $\delta$ the thickness of the diffusion layer, and $v$ the deposition rate in units of velocity. The temperature dependence of the domain diameter gave an activation energy of $0.19 \mathrm{eV}$, yielding an activation energy for diffusion of $0.38 \mathrm{eV}$. Considering measured activation energies for diffusion in the Al-Ge system, this result is consistent with the assumption of a surface interdiffusion mechanism.

The phase separation reported in Ref. 1 was primarily in the film plane, and will be referred to as "lateral." In this letter, we report the observation of a microstructural transition from a laterally phase-separated microstructure to one that is phase separated perpendicular to the film plane ("transverse"), resulting in a two-layer microstructure, as the film thickness increases. This observation is explained by a simple thermodynamic model of the competition of interfacial and surface free energies. We show that restricted kinetics limit the range in which the effect is observed.

Samples of overall composition of $60 \% \mathrm{Al}-40 \% \mathrm{Ge}$ were co-evaporated onto oxidized Si by electron-beam deposition with a base pressure of less than $5 \times 10^{-9}$ Torr. The surface morphology was studied with Hitachi S-800 and S-520 scanning electron microscopes (SEM) at normal and grazing-angle incidence, and the bulk microstructure was observed by transmission electron microscopy (TEM), using a JEOL 2000FX. Cross-sectional SEM samples were prepared by polishing the edge of a substrate-film couple. The phases were identified using $x$-ray diffraction in Siemann-Bohlin geometry and $x$-ray energy dispersive spectroscopy (XEDS) in both 'SEM and TEM. Laterally averaged composition profiles were obtained by Rutherford backscattering spectrometry, using $2 \mathrm{MeV} \mathrm{He}^{+}$ions.

Figure 1 shows SEM micrographs of films deposited at $200^{\circ} \mathrm{C}$, with thicknesses of 110,300 , and $800 \mathrm{~nm}$. For the thinnest film, lateral separation on a scale of $0.3 \mu \mathrm{m}$ is observed. As the thickness increases, the Ge domains appear increasingly covered by Al. A TEM micrograph of the $110 \mathrm{~nm}$ film shows the same lateral separation observed on the surface by SEM. Rutherford backscattering analysis of the $800 \mathrm{~nm}$ film shows that the Ge concentration on the surface and the Al concentration near the substrate approach zero. A direct observation of the separation in the $800 \mathrm{~nm}$ films is provided by the SEM micrographs of a polished edge of the film and substrate in Fig. 2. The emerging picture is one in which an increasing film thickness leads to an increasing degree of transverse separation, with a Ge-rich layer buried under an Al-rich layer. 
(a)

(b)

(c)

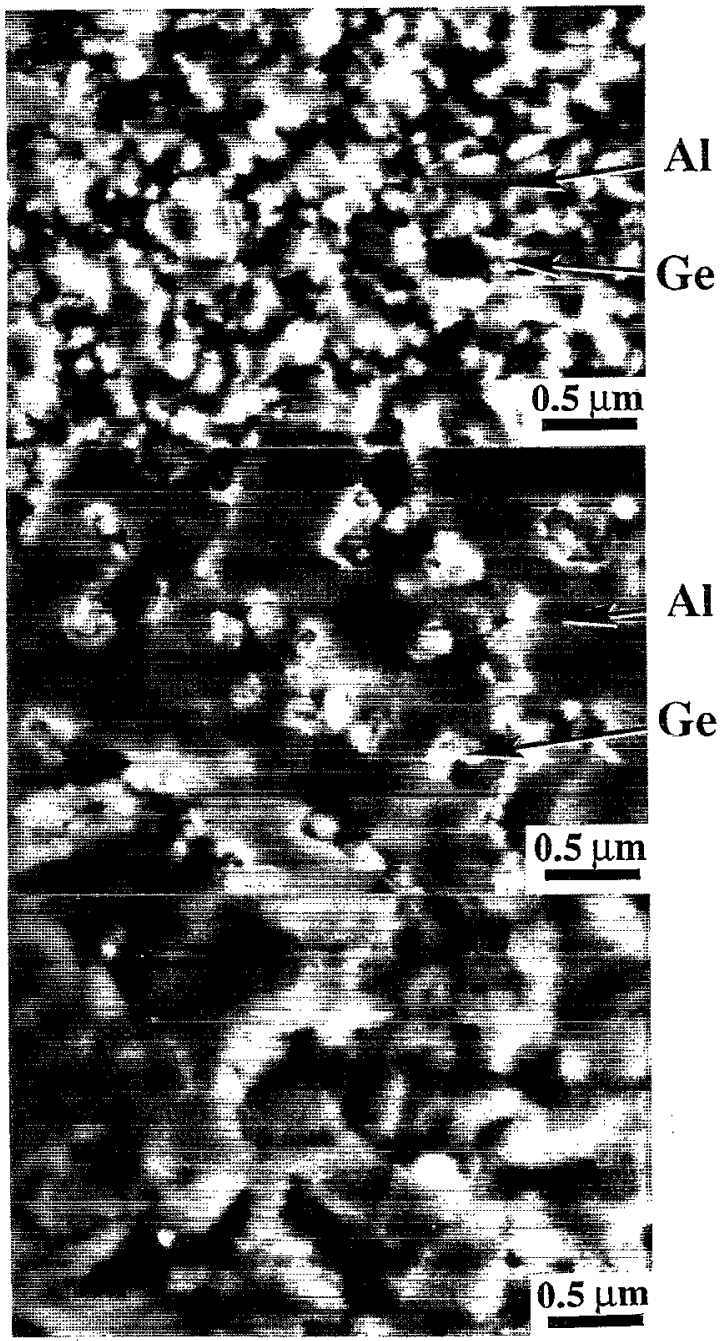

FIG. 1. Secondary electron SEM photomicrographs of films deposited at $200^{\circ} \mathrm{C}$ having thicknesses of (a) $110 \mathrm{~nm}$, (b) $300 \mathrm{~nm}$, and (c) $800 \mathrm{~nm}$. As film thickness increases the Ge-rich domains become increasingly buried beneath and Al-rich overlayer.

In order to analyze this observation, we evaluate the total interfacial energies for idealized models of the observed phase-separated microstructures. For the transverse phasc-separated microstructure, we model the microstructure as two uniform layers, one being Al-rich (denoted by $\alpha$ ), and the other Ge rich (denoted by $\beta$ ). We assume, without loss of generality that the $\alpha$-on- $\beta$ configuration is more stable than $\beta$-on- $\alpha$, i.e.; $\gamma_{\alpha V}+\gamma_{\beta S}<\gamma_{\beta V}+\gamma_{\alpha S}$, where $\gamma_{X Y}$ is the free energy of the $X-Y$ interface, and $X$ and $Y$ represent $\alpha$ and $\beta$ for the two terminal phases, $V$ for the vacuum, and $S$ for the substrate. For lateral separation, the volume fraction of phase $\alpha, x$, is assumed to be smaller than the volume fraction of $\beta(1-x)$, and the $\alpha$ domains are assumed to be cylindrical with radius $R$ and their density $1 / L^{2}$ per unit area, yielding $x=\pi R^{2} / L^{2}$. These simplified phase-separated microstructures are illustrated in Fig. 3. The following expressions are obtained for the total excess free energies per unit film area:
Al

Ge

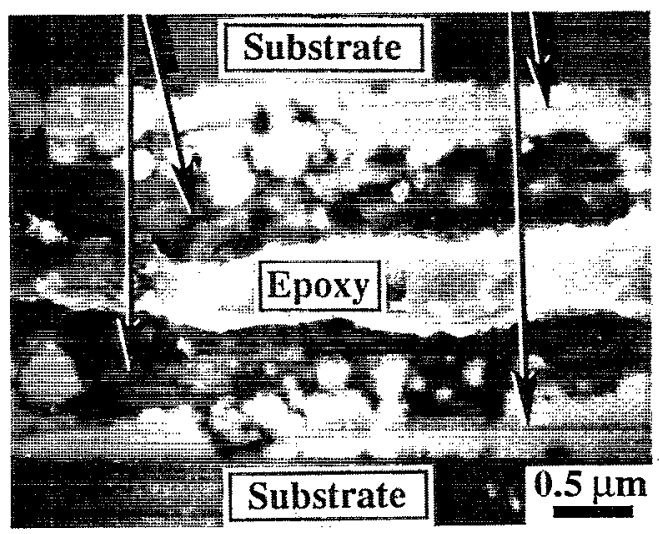

FIG. 2. Secondary electron SEM photomicrograph of a polished cross section through two samples of the $800 \mathrm{~nm}$ thick film deposited at $200^{\circ} \mathrm{C}$. The samples were glued together film-to-film. XEDS shows the film exhibits transverse phase separation with the Al-rich phase at the surface and the Ge-rich phase at the substrate/film interface.

$$
\begin{aligned}
& \gamma_{\text {trans }}=\gamma_{\alpha V}+\gamma_{\alpha \beta}+\gamma_{\beta S}, \\
& \gamma_{\text {lat }}=x\left(\gamma_{\alpha V}+\gamma_{\alpha S}\right)+(1-x)\left(\gamma_{\beta V}+\gamma_{\beta S}\right)+2 \frac{h x}{R} \gamma_{\alpha \beta}
\end{aligned}
$$

where the subscript "trans" stands for transverse and "lat" for lateral phase separation, and $h$ is the film thickness. If $x>1 / 2$, then $x$ in Eq. (1b) should be replaced with $1-x$.
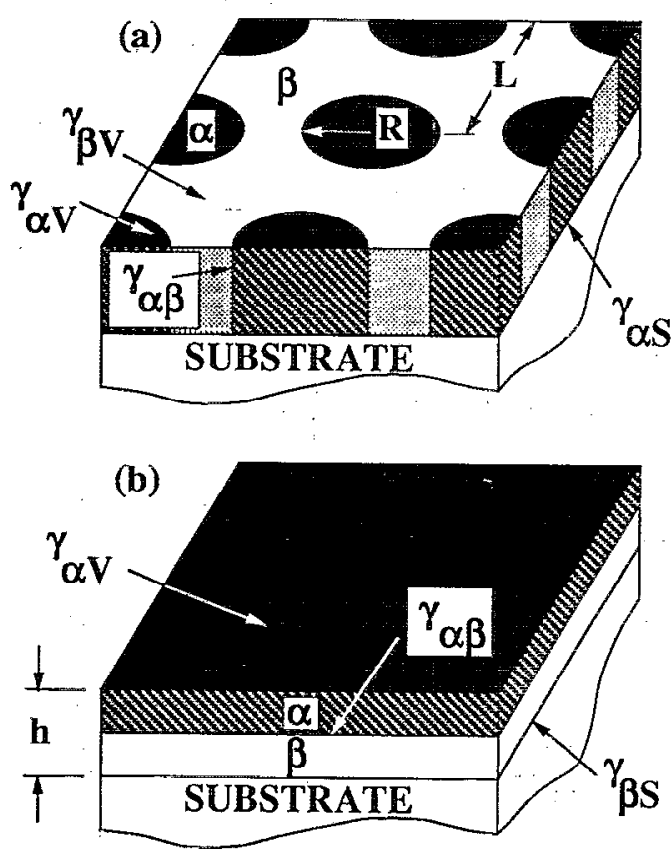

FIG. 3. Sketches of simplified thin film microstructures illustrating (a) lateral phase separation (parallel to the film/subsirate interface) and (b) * transverse phase separation (perpendicular to the film/substrate interface). $\gamma_{X Y}$ is the free energy of the $X-Y$ interface, and $X$ and $Y$ represent: $\alpha$ and $\beta$ for the two terminal phases, $V$ for the vapor, and $S$ for the substrate. 
We can define a critical ratio of film thickness to domain size by equating $\gamma_{\text {trans }}$ and $\gamma_{\text {lat }}$ :

$$
\left[\frac{h}{R}\right]_{c}=\frac{1}{2 x}\left[1+\frac{(1-x)\left(\gamma_{\alpha V}-\gamma_{\beta V}\right)-x\left(\gamma_{\alpha S}-\gamma_{\beta S}\right)}{\gamma_{\alpha \beta}}\right]
$$

so that lateral separation is energetically favored for $[h / R]<[h / R]_{c}$, and transverse separation, for $[h / R]$ $>[h / R]_{c}$. Since the value of $R$ is no greater than its asymptotic limit $R_{\infty}$, the upper bound on the film thickness for which transverse phase separation will occur is $h$ $=R_{\infty}[h / R]_{c}$. If $[h / R]_{c}<0$, then transverse phase separation will be thermodynamically favored from the beginning of film growth. Otherwise, transverse phase separation will become thermodynamically favored after some finite film thickness. It follows that a thermodynamic transition from an initially laterally phase-separated microstructure to a transversely separated one will occur if

$$
x\left(\gamma_{\alpha S}-\gamma_{\beta S}\right)-(1-x)\left(\gamma_{\alpha V}-\gamma_{\beta V}\right)<\gamma_{\alpha \beta} .
$$

For $x=0.5$, the approximate value in our experiments, Eq. (3a) reduces to

$$
\left(\gamma_{\beta V}+\gamma_{\alpha \dot{s}}\right)-\left(\gamma_{\alpha V}+\gamma_{\beta S}\right)<2 \gamma_{\alpha \beta} .
$$

Since the left-hand side of Eq. (3b) is positive (by definition of $\alpha$ and $\beta$, above), we find that $[h / R]_{c}$ is positive only for a sufficiently large $\alpha-\beta$ interfacial energy. For comparison, the criterion for wetting $(\alpha$ on $\beta$ ) is

$$
\gamma_{\alpha \beta}<\gamma_{\beta V}-\gamma_{\alpha V}
$$

Unfortunately, to the best of our knowledge, sufficient interfacial/surface energy data do not exist to validate the thermodynamic predictions. However, our own experiments on deposition of $\mathrm{Al}$ on $\mathrm{Ge}$ and $\mathrm{Ge}$ on $\mathrm{Al}$, as well as several other experimental studies, suggest that $\mathrm{Al}$ wets $\mathrm{Ge}^{4}$ or at least that $\mathrm{Al}$ has lower surface energy than $\mathrm{Ge}^{5}$ This is consistent with our observation here that it is Al which makes up the surface layer in the transverse separated films.

A remaining question is that of the mechanism by which transverse phase separation occurs. As the Al film thickness increases, it is expected to become increasingly difficult to maintain a Ge flux through the growing (Al) layer. Ge-rich domains are then expected to form by one of two possible mechanisms: (i) a Mullins-Sekerka ${ }^{6}$ instability, leading to the formation of Al-rich "fingers" which, in steady state, advance at the same rate as the surface; or (ii) nucleation of Ge-rich domains within the Al-rich phase. Both mechanisms are driven by the supersaturation of the Al-rich phase. Such kinetic processes account for the fact that the ideal layered structure is not observed. The second scenario can be analyzed as follows: let $\Delta C$ be the maxi- mum Ge concentration difference across the Al layer, a quantity which depends on the allowed supersaturation in the (Al) solution. If $d_{\mathrm{Al}}$ is the ( $\mathrm{Al}$ ) layer thickness, then the maximum Ge flux through the (Al) phase is given by

$$
J=-D_{\mathrm{eff}} \Delta C / d_{\mathrm{Al}},
$$

where $D_{\text {eff }}$ is the effective diffusion coefficient of $\mathrm{Ge}$ through the Al layer and may include a combination of bulk and grain boundary diffusivities. When $d_{\mathrm{Al}}$ $>D_{\text {eff }} \Delta C / J_{\mathrm{Ge}}^{\mathrm{dep}}$, where $J_{\mathrm{Ge}}^{\mathrm{dep}}$ is the deposition flux of germanium vapor, complete transverse separation cannot be maintained. In this case, we expect nucleation of a Ge-rich phase within the Al-rich layer, leading again to lateral separation, while maintaining a steady-state Al-rich layer of approximate thickness $D \Delta C / J_{\mathrm{Ge}}^{\mathrm{dep}}$ near the surface. This is consistent with our observations for films deposited at 70 $150^{\circ} \mathrm{C}$, for which Rutherford backscattering spectra show $\mathrm{Ge}$ depletion and $\mathrm{Al}$ enhancement near the surface. At these temperatures, the diffusion distance, $D \Delta C / J_{\mathrm{Ge}}^{\mathrm{dep}}$, is significantly smaller than the film thickness of $100-200 \mathrm{~nm}$. The bulk microstructure in these samples is indeed uniform with evenly distributed domains.

In summary, we have observed a transition in the phase-separated microstructure in co-deposited Al-Ge films. Lateral separation gives way to transverse separation as the film grows thicker. A thermodynamic analysis of the surface and interfacial energies shows that such a transition is expected for any phase-separated system. However, the scale of the transverse phase separation is kinetically limited.

This project was partly funded by the National Science Foundation, grant No. DMR-8820285, and the US Air Force Office of Scientific Research, Contract AFOSR-900112. Rutherford Backscattering Spectrometry experiments were performed in the Michigan Ion Beam Laboratory at the University of Michigan.

${ }^{1}$ C. D. Adams, M. Atzmon, Y.-T. Cheng, and D. J. Srolovitz, in Thin Film Structures and Phase Stability, edited by B. M. Clemens and W. L. Johnson, Mater. Res. Soc. Symp. Proc., Vol. 187 (Materials Research Society, Pittsburgh, 1990), p. 33.

${ }^{2}$ M. Atzmon, C. D. Adams, Y.-T. Cheng, and D. J. Srolovitz, in Evolution of Thin Film and Surface Microstructure, edited by C. V. Thompson, J. Y. Tsao, and D. J. Srolovitz, Mater. Res. Soc. Symp. Proc., Vol. 202 (Materials Research Society, Pittsburgh, 1991), p. 143.

${ }^{3}$ Binary Alloy Phase Diagrams, edited by T. B. Massalski (ASM, MetalsPark, 1986), p. 116.

${ }^{4}$ B. Dwir and G. Deutscher, in Proceedings of International Workshop on Novel Mechanism of Superconductivity, edited by S. A. Wolf and V.Z. Kresin (Plenum, NY, 1987), p. 23.

${ }^{5}$ S. S. Lau and W. F. van der Wege, in Thin Films-Interdiffusion and Reactions, edited by J. M. Poate, K. N. Tu, and J. W. Mayer (Wiley, New York, 1978), p. 433.

${ }^{6}$ W. W. Mullins and R. F. Sekerka, J. Appl. Phys. 34, 323 (1963); ibid. 35, 444 (1964). 